Received: 08.04.2021

Revised: 19.06.2021

Accepted: 25.06 .2021

DOI: $10.17804 / 2410-9908.2021 .3 .055-070$

\title{
ANALYSIS OF SPECIFIC KINETIC ENERGY FOR THE BIRIKH-OSTROUMOV SHEAR DIFFUSION FLOW
}

\author{
N. V. Burmasheva ${ }^{\text {a) }}$ and E. Yu. Prosviryakov ${ }^{\text {b) }}$ \\ Institute of Engineering Science, Ural Branch of the Russian Academy of Sciences, \\ 34 Komsomolskaya St., Ekaterinburg, 620049, Russian Federation \\ a) (iD https://orcid.org/0000-0003-4711-1894 $\otimes$ nat_burm@mail.ru; \\ b) (iD https://orcid.org/0000-0002-2349-7801 《evgen_pros@mail.ru \\ *Corresponding author. E-mail: nat_burm@mail.ru \\ Address for correspondence: 34 Komsomolskaya St., Ekaterinburg, 620049, Russian Federation \\ Tel.:+7 (343) 37535 76; fax: 3745330
}

The article presents a new exact solution for stratified steady-state shear diffusion flows of a viscous fluid in an infinite horizontal layer with impenetrable boundaries. The announced exact solution belongs to the Ostroumov-Birikh family. Two components of the velocity vector depend on the vertical (transverse) coordinate. The concentration field and the pressure field are described by linear forms relative to horizontal (longitudinal) coordinates, with coefficients depending on the third coordinate. The components of the velocity field and the shear stress field are analyzed in detail, and the behavior of the specific kinetic energy is studied. It is shown that this exact solution is capable of describing the stratification of the shear stress field and the nonmonotonic behavior of flow velocity. The relation of flow velocities and shear stresses to the distribution of specific kinetic energy is revealed.

Keywords: concentration convection, Navier-Stokes equation, exact solution, shear flow, stratification.

\section{References}

1. Zuev A.L., Kostarev K.G. Experimental study of the features of concentration-capillary convection. Vestnik Permskogo nauchnogo tsentra UrO RAN, 2009, no. 4, pp. 4-15. (In Russian).

2. $\quad$ Birikh R.V., Mazunina E.S., Mizev A.I., Rudakov R.N. Solutal convection induced by submerged source of surface-active substance. Konvektivnye Techeniya, 2009, no. 4, pp. 063-084. (In Russian).

3. Evstratova K.I., Kupina N.A., Malakhova E.E. Fizicheskaya i kolloidnaya khimiya [Physical and colloidal chemistry]. Moscow, Vysshaya shkola, 1990, 487 p. (In Russian).

4. Petrov N.Kh. A fluorescence spectroscopy study of preferential solvation in binary solvents. High Energy Chemistry, 2006, vol. 40, no. 1, pp. 22-34. DOI: 10.1134/S001814390601005X.

5. Gurevich A.E., Kapchenko L.N., Kruglikov N.M. Teoreticheskiye osnovy neftyanoy gidrogeologii [Theoretical foundations of petroleum hydrogeology]. Leningrad, Nedra Publ., 1972, 272 p. (In Russian).

6. Petrov T.G., Treyvus E.B., Kasatkin A.P. Vyrashchivaniye kristallov iz rastvorov [Growing crystals from solutions]. Leningrad, Nedra Publ., 1983, 200 p. (In Russian).

7. Kartsev A.A. Gidrogeologiya neftyanykh i gazovykh mestorozhdeniy [Hydrogeology of oil and gas fields, 2nd edition, rev. and add.]. Moscow, Nedra Publ., 1972, 280 p. (In Russian).

8. Aristov S.N., Prosviryakov E.Yu., Spevak L.F. Nonstationary laminar thermal and solutal Marangoni convection of a viscous fluid. Vychislitel'naya mekhanika sploshnykh sred [Computa- 
tional Continuum Mechanics], 2015, vol. 8, no. 4, pp. 445-456. DOI: 10.7242/19996691/2015.8.4.38.27. (In Russian).

9. Beschastnov M.V., Sokolov V.M., Katz M.I. Avarii v khimicheskikh proizvodstvakh i mery ikh preduprezhdeniya [Accidents in chemical industries and measures for their prevention]. Moscow, Khimiya Publ., 1976, 368 p. (In Russian).

10. Pryanikov V.I. Tekhnika bezopasnosti v khimicheskoy promyshlennosti [Safety in the chemical industry]. Moscow, Khimiya Publ., 1989, 288 p. (In Russian).

11. Surguchev M.L., Zheltkov Yu.V., Simkin E.M. Fiziko-khimicheskiye mikroprotsessy $v$ neftegazonosnykh plastakh [Physicochemical microprocesses in the oil and gas-bearing formations]. Moscow, Nedra Publ., 1984, 215 p. (In Russian).

12. Walmsley H.L. The calculation of the electrostatic potentials that occur when tanks are filled with charged liquids. J. Electrostatics, 1991, vol. 26, pp. 201-226. DOI: 10.1016/03043886(91)90016-9.

13. Walmsley H.L. Threshold potentials and discharge charge transfers for the evaluation of electrostatic hazards in road -tanker loading. J. Electrostatics, 1991, vol. 26, pp. 157-174. DOI: 10.1016/0304-3886(91)90013-6.

14. Walmsley H.L., Mills J.S. Electrostatic ignition hazards in road tanker loading: Part 1. J. Electrostatics, 1992, vol. 28, pp. 61-88. DOI: 10.1016/0304-3886(92)90028-R.

15. Walmsley H.L., Gregory K.E. Electrostatic ignition hazards in road tanker loading: Part 2. J. Electrostatics, 1992, vol. 28, pp. 99-124. DOI: 10.1016/0304-3886(92)90065-2.

16. Walmsley H.L., Gregory K.E. Electrostatic ignition hazards in road tanker loading: Part 3. J. Electrostatics, 1992, vol. 28, pp. 125-148. DOI: 10.1016/0304-3886(92)90066-3.

17. Fung P., Chen H., Touchard G.G., Radke C.J. A nonlinear corrosion double layer model for laminar flow electrification of hydrocarbon liquids in long metal pipes. J. Electrostatics, 1997, vol. 40, pp. 45-54. DOI: 10.1016/S0304-3886(97)00013-2.

18. Gershuni G.Z., Zhukhovitskii E.M. Convective stability of incompressible fluids. Israel Program for Scientific Translations, Jerusalem, Keter Publishing House, 1976, 330 p.

19. Ryzhkov I.I., Stepanova I.V. Group properties and exact solutions of equations for vibrational convection of a binary mixture. Journal of Applied Mechanics and Technical Physics, 2011, vol. 52, no. 4, pp. 560-570. DOI: 10.1134/S0021894411040080.

20. Burmasheva N.V., Prosviryakov E.Yu. Exact solution for stable convective concentration flows of a Couette type. Vychislitel'naya mekhanika sploshnykh sred [Computational Continuum Mechanics], 2020, vol. 13, no. 3, pp. 337-349. DOI: 10.7242/1999-6691/2020.13.3.27. (In Russian).

21. Lin C.C. Note on a class of exact solutions in magneto-hydrodynamics. Arch. Rational Mech. Anal., 1957, vol. 1, pp. 391-395. DOI: 10.1007/BF00298016.

22. Sidorov A.F. Two classes of solutions of the fluid and gas mechanics equations and their connection to traveling wave theory. J. Appl. Mech. Tech. Phys., 1989, vol. 30, no. 2, p. 197-203. DOI: $10.1007 / \mathrm{BF} 00852164$.

23. Aristov S.N., Prosviryakov E.Y. A new class of exact solutions for three-dimensional thermal diffusion equations. Theor. Found. Chem. Eng., 2016, vol. 50, pp. 286-293. DOI: $10.1134 / \mathrm{S} 0040579516030027$.

24. Burmasheva N.V., Prosviryakov E.Yu. On Marangoni shear convective flows of inhomogeneous viscous incompressible fluids in view of the Soret effect. Journal of King Saud UniversityScience, 2020, vol. 32, iss. 8, pp. 3364-3371. DOI: 10.1016/j.jksus.2020.09.023.

25. Knyazev D.V. Two-dimensional flows of a viscous binary fluid between moving solid boundaries. Journal of Applied Mechanics and Technical Physics, 2011, vol. 52, no. 2, pp. 212-217. DOI: $10.1134 / \mathrm{S} 0021894411020088$.

26. Ostroumov G.A. Free convection under the condition of the internal problem. Washington, NACA Technical Memorandum 1407, National Advisory Committee for Aeronautics, 1958. 
27. Birikh R.V. Thermocapillary convection in a horizontal layer of liquid. J. Appl. Mech. Tech. Phys., 1966, no. 7, p. 43-44.

28. Su T.C. Obtaining the exact solutions of the Navie-Stokes equations. International Journal of Non-linear Mechanics, 1985, vol. 20, no. 1, pp. 9-19.

29. Petrov A.G. Exact solution of the Navier-Stokes equations in a fluid layer between the moving parallel plates. Journal of Applied Mechanics and Technical Physics, 2012, vol. 53, no, 5, pp. 642-646. DOI: 10.1134/S0021894412050021.

30. Pukhnachev V.V. Symmetries in the Navier-Stokes equations. Uspekhi matematiki, 2006, vol. 4, no. 1, pp. 6-76. (In Russian).

31. Riabouchinsky D. Quelques considerations sur les mouvements plans rotationnels d' un liquid. C. R. Hebdomadaires Acad. Sci., 1924, vol. 179, pp. 1133-1136.

32. Pukhnachev V.V. Group properties of the Navier-Stokes equations in the plane case. Prikladnaya Mekhanika i Tekhnicheskaya Fizika, 1960, no. 1, pp. 83-90. (In Russian).

33. Aristov S.N., Knyazev D.V., Polyanin A.D. Exact solutions of the Navier-Stokes equations with the linear dependence of velocity components on two space variables. Theoretical Foundations of Chemical Engineering, 2009, vol. 43, no. 5, pp. 642-662. DOI: 10.1134/S0040579509050066.

34. Prosviryakov E.Yu. Exact solutions of three-dimensional potential and vortical Couette flows of a viscous incompressible fluid. Vestnik NIYaU MIFI, 2015, vol. 4, no. 6, pp. 501-506. DOI: 10.1134/S2304487X15060127. (In Russian).

35. Polyanin A.D., Zhurov A.I. Integration of linear and some model non-linear equations of motion of incompressible fluids. International Journal of Non-Linear Mechanics, 2013, vol. 49, pp. 77-83. DOI: 10.1016/j.ijnonlinmec.2012.08.004.

36. Ludlow D.K., Clarkson P.A., Bassom A. Nonclassical symmetry reductions of the threedimensional incompressible Navier-Stokes equations. Journal of Physics A General Physics, 1999, vol. 31, no. 39, 7965. DOI: 10.1088/0305-4470/31/39/012.

37. Aristov S.N., Polyanin A.D. Exact solutions of unsteady three-dimensional Navier-Stokes equations. Doklady Physics, 2009, vol. 54, no. 7, pp. 316-321. DOI: 10.1134/S1028335809070039. 38. Pukhnachev V.V. Symmetries in the Navier-Stokes equations. Uspekhi Mekhaniki, 2006, no. 6, pp. 3-76. (In Russian).

39. Kuznetsova Ju.L., Skul'skiy O.I., Pyshnograi G.V. Presure driven flow of a nonlinear viscoelastic fluid in a plane channel. Vychislitel'naya mekhanika sploshnykh sred [Computational Continuum Mechanics], 2010, vol. 3, no. 2, pp. 55-69. (In Russian).

40. Aristov S.N., Skulsky O.I. Exact solution of the problem of flow of a six-constant model of Jeffreys fluid in a plane channel. Prikladnaya Mekhanika i Tekhnicheskaya Fizika, 2002, vol. 43, no. 6, pp. 39-45. (In Russian).

41. Knyazev D.V., Kolpakov I.Yu. The exact solutions of the problem of a viscous fluid flow in a cylindrical domain with varying radius. Nelineynaya Dinamika [Russian Journal of Nonlinear Dynamics], 2015, vol. 11, no. 1, pp. 89-97.

42. Goncharova O.N., Rezanova E.V., Lyulin Y.V., Kabov O.A. Analysis of a convective fluid flow with a concurrent gas flow with allowance for evaporation. High Temperature, 2017, vol. 55, no. 6, pp. 887-897. DOI: 10.1134/S0018151X17060074.

43. Burmasheva N.V., Prosviryakov E.Yu. A large-scale layered stationary convection of an incompressible viscous fluid under the action of shear stresses at the upper boundary. Velocity field investigation. Vestnik SamGTU. Seriya fiziko-matematicheskie nauki [Journal of Samara State Technical University, Ser. Physical and Mathematical Sciences], 2017, vol. 21, no. 1, pp. 180-196. DOI: 10.14498/vsgtu1527. (In Russian).

44. Burmasheva N.V., Prosviryakov E.Yu. Temperature field investigation in layered flows of a vertically swirling viscous incompressible fluid under two thermocapillar forces at a free boundary. Diagnostics, Resource and Mechanics of materials and structures, 2019, iss. 1, pp. 6-42. DOI: 10.17804/2410-9908.2019.1.006-042. 
45. Burmasheva N.V., Larina E.A., Prosviryakov E.Yu. Unidirectional convective flows of a viscous incompressible fluid with slippage in a closed layer. AIP Conference Proceedings, 2019, vol. 2176, 030023. DOI: 10.1063/1.5135147.

46. Burmasheva N.V., Prosviryakov E.Yu., Thermocapillary convection of a vertical swirling liquid. Theoretical Foundations of Chemical Engineering, 2020, vol. 54, no. 1, pp. 230-239. DOI: $10.1134 / \mathrm{S} 0040579519060034$.

47. Burmasheva N.V., Prosviryakov E.Yu. Exact solution of Navier-Stokes equations describing spatially inhomogeneous flows of a rotating fluid. Trudy Instituta Matematiki i Mekhaniki UrO $R A N$, 2020, vol. 26, no. 2, pp. 79-87. DOI: 10.21538/0134-4889-2020-26-2-79-87. (In Russian).

48. Burmasheva N.V., Larina E.A., Prosviryakov E.Yu. Unidirectional convective flow of viscous incompressible fluid in a closed horizontal layer with the perfect slip condition. AIP Conference Proceedings, 2020, vol. 2315, 020010. DOI: 10.1063/5.0036714. (In Russian).

49. Burmasheva, N.V., Privalova, V.V., Prosviryakov, E.Y. Layered Marangoni convection with the Navier slip condition. Sādhanā, 2021, vol. 46, 55. DOI: 10.1007/s12046-021-01585-5.

50. Burmasheva N.V., Prosviryakov E.Yu. Analysis of non-one-dimensional shear concentration convective flows of a viscous incompressible fluid in a plane horizontal layer with motionless boundaries. AIP Conference Proceedings, 2020, vol. 2315, 020007. DOI: 10.1063/5.0036710.

51. Sedov L.I. Continuum Mechanics, vol. 1. Moscow, Nauka Publ., 1970, 492 p. (In Russian).

52. Truesdell C. Pervonachalnyi kurs ratsionalnoy mekhaniki sploshnykh sred [A First Course in Rational Continuum Mechanics, Engl. transl.]. Moscow, Mir Publ., 1975. (In Russian).

53. Vallander S.V. Lektsii po gidroaeromekhanike [Lectures on hydroaeromechanics]. Leningrad, Izdatel'stvo Leningradskogo universiteta Publ., 1978. (In Russian).

54. Pokrovsky V.N. Statisticheskaya mekhanika razbavlennykh suspenziy [Statistical mechanics of dilute suspensions]. Moscow, Nauka Publ., 1978. (In Russian). 
Подана в журнал: 08.04.2021

УДК 51-72

DOI: $10.17804 / 2410-9908.2021 .3 .055-070$

\title{
АНАЛИЗ УДЕЛЬНОЙ КИНЕТИЧЕСКОЙ ЭНЕРГИИ В СДВИГОВОМ ДИФФУЗИОННОМ ТЕЧЕНИИ БИРИХА-ОСТРОУМОВА
}

\author{
Н. В. Бурмашева ${ }^{\text {a)* }}$, Е. Ю. Просвиряков ${ }^{\text {) }}$ \\ Институт машиноведения УрО РАН, \\ 34, г. Екатеринбург, Российская Федерация \\ a) iD https://orcid.org/0000-0003-4711-1894 nat_burm@mail.ru; \\ б) iD https://orcid.org/0000-0002-2349-7801 《 evgen_pros@mail.ru \\ *Ответственный автор. Электронная почта: nat_burm@mail.ru \\ Адрес для переписки: ул. Комсомольская, 34, г. Екатеринбург, Российская Федерация \\ Тел.: +7 (343) 375-35-76; факс: 374-53-30
}

В статье представлено новое точное решение для слоистых установившихся сдвиговых диффузионных течений вязкой жидкости в бесконечном горизонтальном слое с непроницаемыми границами. Анонсируемое точное решение принадлежит семейству Остроумова-Бириха. Две компоненты вектора скорости зависят от вертикальной (поперечной) координаты. Поле концентрации и поле давления описываются линейными формами относительно горизонтальных (продольных) координат с коэффициентами, зависящими от третьей координаты. Проведен подробный анализ компонент поля скорости и поля касательных напряжений, изучен характер поведения удельной кинетической энергии. Показано, что данное точное решение способно описывать расслоение поля касательных напряжений и немонотонный характер изменения скорости течения. Выявлена связь скоростей течения и касательных напряжений с распределением удельной кинетической энергии.

Ключевые слова: концентрационная конвекция, уравнение Навье-Стокса, точное решение, сдвиговое течение, стратификация.

\section{1. Введение}

Концентрационная конвекция возникает в результате повышенной минерализации одних слоев жидкости по сравнению с уровнем минерализации других ее слоев. Этот вид конвекции наиболее сильно проявляется для рассолов поверхностных водоемов, солончаков, соленосных пород и других природных явлений и техногенных факторов [1-8]. Однако концентрационная конвекция возникает не только в природных водоемах, но и в технических устройствах вследствие неоднородности распределения различного рода примеси в рабочей жидкости. Распределение примеси при этом существенно влияет на свойства потока жидкости, его вязкость, среднюю скорость течения, возможность возникновения зон с обратным течением.

Возможность управления противотечениями оказывается особенно важна в технических устройствах, поскольку наблюдаемый при некоторых сочетаниях задаваемых параметров эффект попеременного ослабления и усиления скоростей жидкости относительно стенок может приводить к увеличению электризации жидкости в насосах, расходомерах и других подобных устройствах [9-17]. В предельном случае электризация может привести к воспламенению горючих сред искровыми разрядами с электростатически заряженных материалов.

Статья посвящена сдвиговому диффузионному течению вязкой несжимаемой жидкости в горизонтальном слое, вызванному неравномерным распределением концентрации примеси; приведено новое точное решение системы уравнений концентрационной конвекции 
с учетом прилипания жидкости к твердым непроницаемым стенкам, ограничивающим слой. Основное внимание уделено анализу свойств поля скоростей течения. Показано, что приведенное точное решение обеспечивает отсутствие зон с обратным течением, при этом характеристики течения (кинетическая энергия, средняя скорость и др.) зависят от распределения примеси на одной из границ рассматриваемого слоя.

\section{2. Постановка задачи}

Рассматривается краевая задача, описывающая сдвиговое установившееся диффузионное течение вязкой несжимаемой жидкости в бесконечном горизонтальном слое толщины $h$. В определяющую систему уравнений входят уравнение Навье-Стокса, уравнения несжимаемости и уравнение изменения концентрации легкой фазы бинарной смеси [18-20]:

$$
\begin{gathered}
(\boldsymbol{V} \cdot \nabla) \boldsymbol{V}=-\nabla P+v \Delta \boldsymbol{V}+g \beta C \boldsymbol{k} \\
(\boldsymbol{V} \cdot \nabla) C=d \Delta C ; \\
\nabla \cdot \boldsymbol{V}=0 .
\end{gathered}
$$

Здесь $\boldsymbol{V}=\left(V_{x}(x, y, z), V_{y}(x, y, z), 0\right)-$ вектор скорости; $P(x, y, z)-$ отклонение от гидростатического давления, деленное на среднюю плотность жидкости; $C(x, y, z)$ - отклонение концентрации легкой фазы смеси от равновесного значения. Параметры $v, d-$ кинематическая (молекулярная) вязкость жидкости и коэффициент диффузии соответственно; $g$ - ускорение свободного падения; $\beta$ - коэффициент концентрационного расширения, $\boldsymbol{k}$ - орт вертикальной оси $O z$. [20-25]:

Решение системы уравнений концентрационной конвекции (1) ищем в классе Линя

$$
\begin{gathered}
V_{x}=U(z) ; V_{y}=V(z) ; V_{z}=0 \\
P=P_{0}(z)+x P_{1}(z)+y P_{2}(z) ; C=C_{0}(z)+x C_{1}(z)+y C_{2}(z) .
\end{gathered}
$$

В результате допущений (2) и (3) относительно установившихся гидродинамических полей система уравнений Обербека-Буссинеска редуцируется к следующей эквивалентной системе линейных обыкновенных дифференциальных уравнений с постоянными коэффициентами:

$$
\begin{gathered}
C_{1}{ }^{\prime \prime}=0 ; \quad C_{2}{ }^{\prime \prime}=0 ; \\
P_{1}^{\prime}=g \beta C_{1} ; \quad P_{2}^{\prime}=g \beta C_{2} ; \\
v V^{\prime \prime}=P_{2}, \quad v U^{\prime \prime}=P_{1}, \\
d C_{0}^{\prime \prime}=U C_{1}+V C_{2}, \\
P_{0}^{\prime}=g \beta C_{0} .
\end{gathered}
$$

Штрихом в системе (4) обозначена операция дифференцирования по переменной $z$. Системы дифференциальных уравнений, подобные системе (4), также исследовались различными авторами для термодиффузионных задач и задач тепловой и концентрационной конвекции [8, 19, 20, 23-49]. 
Общее решение системы (4) получаем последовательным интегрированием. Сначала определяется зависимость горизонтальных градиентов от вертикальной координаты:

$$
C_{1}=c_{1} z+c_{2} ; C_{2}=c_{3} z+c_{4}
$$

Далее однократным интегрированием получено распределение горизонтальных градиентов давления по толщине слоя:

$$
P_{1}=g \beta\left(c_{1} \frac{z^{2}}{2}+c_{2} z+c_{5}\right) ; P_{2}=g \beta\left(c_{3} \frac{z^{2}}{2}+c_{4} z+c_{6}\right)
$$

Скорости течения жидкости описываются многочленами четвертой степени:

$$
U=\frac{g \beta}{v}\left(c_{1} \frac{z^{4}}{4 !}+c_{2} \frac{z^{3}}{3 !}+c_{5} \frac{z^{2}}{2}+c_{7} z+c_{8}\right) ; \quad V=\frac{g \beta}{v}\left(c_{3} \frac{z^{4}}{4 !}+c_{4} \frac{z^{3}}{3 !}+c_{6} \frac{z^{2}}{2}+c_{9} z+c_{10}\right)
$$

Для вычисления фоновой концентрации $C_{0}$ подставим выражения для скоростей и горизонтальных градиентов концентрации:

$$
\begin{gathered}
d C_{0}{ }^{\prime \prime}=U C_{1}+V C_{2}=\frac{g \beta}{v}\left(c_{1} \frac{z^{4}}{4 !}+c_{2} \frac{z^{3}}{3 !}+c_{5} \frac{z^{2}}{2}+c_{7} z+c_{8}\right)\left(c_{1} z+c_{2}\right)+ \\
+\frac{g \beta}{v}\left(c_{3} \frac{z^{4}}{4 !}+c_{4} \frac{z^{3}}{3 !}+c_{6} \frac{z^{2}}{2}+c_{9} z+c_{10}\right)\left(c_{3} z+c_{4}\right)= \\
=\frac{g \beta}{v}\left(c_{1}^{2} \frac{z^{5}}{4 !}+c_{1} c_{2} \frac{z^{4}}{3 !}+c_{1} c_{5} \frac{z^{3}}{2}+c_{1} c_{7} z^{2}+c_{1} c_{8} z\right)+\frac{g \beta}{v}\left(c_{1} c_{2} \frac{z^{4}}{4 !}+c_{2}^{2} \frac{z^{3}}{3 !}+c_{2} c_{5} \frac{z^{2}}{2}+c_{2} c_{7} z+c_{2} c_{8}\right)+ \\
+\frac{g \beta}{v}\left(c_{3}^{2} \frac{z^{5}}{4 !}+c_{3} c_{4} \frac{z^{4}}{3 !}+c_{3} c_{6} \frac{z^{3}}{2}+c_{3} c_{9} z^{2}+c_{3} c_{10} z\right)+\frac{g \beta}{v}\left(c_{3} c_{4} \frac{z^{4}}{4 !}+c_{4}^{2} \frac{z^{3}}{3 !}+c_{4} c_{6} \frac{z^{2}}{2}+c_{4} c_{9} z+c_{4} c_{10}\right)= \\
=\frac{g \beta}{v}\left(5 c_{1}^{2} \frac{z^{5}}{5 !}+4 c_{1} c_{2} \frac{z^{4}}{4 !}+3 c_{1} c_{5} \frac{z^{3}}{3 !}+2 c_{1} c_{7} \frac{z^{2}}{2 !}+c_{1} c_{8} z\right)+\frac{g \beta}{v}\left(c_{1} c_{2} \frac{z^{4}}{4 !}+c_{2}^{2} \frac{z^{3}}{3 !}+c_{2} c_{5} \frac{z^{2}}{2}+c_{2} c_{7} z+c_{2} c_{8}\right)+ \\
+\frac{g \beta}{v}\left(5 c_{3}^{2} \frac{z^{5}}{5 !}+4 c_{3} c_{4} \frac{z^{4}}{4 !}+3 c_{3} c_{6} \frac{z^{3}}{3 !}+2 c_{3} c_{9} \frac{z^{2}}{2 !}+c_{3} c_{10} z\right)+\frac{g \beta}{v}\left(c_{3} c_{4} \frac{z^{4}}{4 !}+c_{4}^{2} \frac{z^{3}}{3 !}+c_{4} c_{6} \frac{z^{2}}{2 !}+c_{4} c_{9} z+c_{4} c_{10}\right)= \\
=\frac{g \beta}{v}\left[5\left(c_{1}^{2}+c_{3}^{2}\right) \frac{z^{5}}{5 !}+5\left(c_{1} c_{2}+c_{3} c_{4}\right) \frac{z^{4}}{4 !}+\left(3 c_{1} c_{5}+3 c_{3} c_{6}+c_{2}^{2}+c_{4}^{2}\right) \frac{z^{3}}{3 !}+\right. \\
\left.+\left(2 c_{1} c_{7}+2 c_{3} c_{9}+c_{2} c_{5}+c_{4} c_{6}\right) \frac{z^{2}}{2 !}+\left(c_{1} c_{8}+c_{3} c_{10}+c_{2} c_{7}+c_{4} c_{9}\right) z+c_{2} c_{8}+c_{4} c_{10}\right] .
\end{gathered}
$$

Тривиальные выкладки, проведенные выше, даны для лучшего понимания физического смысла последующих формул и полноты исследования математических особенностей точных решений уравнений Обербека-Буссинеска. Выражение для функции $C_{0}$ после интегрирования последнего обыкновенного дифференциального уравнения второго порядка запишем следующим образом: 


$$
\begin{gathered}
C_{0}=\frac{g \beta}{d v}\left[5\left(c_{1}^{2}+c_{3}^{2}\right) \frac{z^{7}}{7 !}+5\left(c_{1} c_{2}+c_{3} c_{4}\right) \frac{z^{6}}{6 !}+\left(3 c_{1} c_{5}+3 c_{3} c_{6}+c_{2}^{2}+c_{4}^{2}\right) \frac{z^{5}}{5 !}+\right. \\
\left.+\left(2 c_{1} c_{7}+2 c_{3} c_{9}+c_{2} c_{5}+c_{4} c_{6}\right) \frac{z^{4}}{4 !}+\left(c_{1} c_{8}+c_{3} c_{10}+c_{2} c_{7}+c_{4} c_{9}\right) \frac{z^{3}}{3 !}+\left(c_{2} c_{8}+c_{4} c_{10}\right) \frac{z^{2}}{2 !}+c_{11} z+c_{12}\right]
\end{gathered}
$$

Для завершения записи точного общего решения системы уравнений (4) приведем выражение для фонового давления:

$$
\begin{gathered}
P_{0}=\frac{g^{2} \beta^{2}}{d v}\left[5\left(c_{1}^{2}+c_{3}^{2}\right) \frac{z^{8}}{8 !}+5\left(c_{1} c_{2}+c_{3} c_{4}\right) \frac{z^{7}}{7 !}+\left(3 c_{1} c_{5}+3 c_{3} c_{6}+c_{2}^{2}+c_{4}^{2}\right) \frac{z^{6}}{7 !}+\right. \\
\left.+\left(2 c_{1} c_{7}+2 c_{3} c_{9}+c_{2} c_{5}+c_{4} c_{6}\right) \frac{z^{5}}{5 !}+\left(c_{1} c_{8}+c_{3} c_{10}+c_{2} c_{7}+c_{4} c_{9}\right) \frac{z^{4}}{4 !}+\left(c_{2} c_{8}+c_{4} c_{10}\right) \frac{z^{3}}{3 !}+c_{11} \frac{z^{2}}{2 !}+c_{12} z+c_{13}\right] .
\end{gathered}
$$

Таким образом, получен набор формул для описания диффузионной конвекции, принадлежащих классу многочленов. Эти формулы сравнительно простые, но, как будет показано далее, содержат ряд интересных физически содержательных результатов.

\section{3. Краевая задача и ее точное решение}

В качестве краевых условий взяты следующие возмущения гидродинамических полей на обеих плоских границах бесконечно протяженной горизонтальной полосы. Нижняя граница слоя жидкости $z=0$ считается гидрофильной (жидкость прилипает) и непроницаемой для растворенного вещества:

$$
U(0)=V(0)=0,\left.C_{0}{ }^{\prime}\right|_{z=0}=\left.C_{1}{ }^{\prime}\right|_{z=0}=\left.C_{2}{ }^{\prime}\right|_{z=0}=0
$$

На верхней неподвижной границе $z=h$ задано однородное давление, отвечающее атмосферному, неоднородное распределение концентрации примеси (горизонтальные градиенты) и условие прилипания:

$$
U(h)=V(h)=0 ;\left.P\right|_{z=h}=S_{0} ; C_{0}(h)=0 ; C_{1}(h)=a ; \quad C_{2}(h)=b .
$$

Решение краевой задачи (4)-(6) имеет следующий вид [50]:

$$
\begin{gathered}
U=\psi Z(-2+Z)(-1+Z) ; \\
V=\gamma Z(-2+Z)(-1+Z) ; \\
C_{1}=a ; C_{2}=b ; \\
C_{0}=\lambda(-1+Z)\left(8+8 Z+8 Z^{2}-12 Z^{3}+3 Z^{4}\right) ; \\
P_{1}=\xi_{1}(-1+Z) ; P_{2}=\xi_{2}(-1+Z) ; \\
P_{0}=S_{0}+\xi_{3}(-1+Z)^{2}\left(11+6 Z+Z^{2}-4 Z^{3}+Z^{4}\right) .
\end{gathered}
$$

В выражениях (7) для удобства дальнейшего анализа и наглядности введены следующие обозначения: 


$$
\begin{gathered}
Z=\frac{z}{h} ; \psi=\frac{a g \beta h^{3}}{6 v} ; \gamma=\frac{b g \beta h^{3}}{6 v} ; \lambda=\left(a^{2}+b^{2}\right) \frac{g h^{5} \beta}{360 d v} \\
\xi_{1}=a g \beta h ; \quad \xi_{2}=b g \beta h ; \xi_{3}=\left(a^{2}+b^{2}\right) \frac{g^{2} h^{6} \beta^{2}}{720 d v}
\end{gathered}
$$

Решение (7) является обобщением решения Пуазейля на неодномерный случай диффузионных течений. Далее подробно проанализированы неодномерное поле скоростей течения, поле касательных напряжений, им порождаемое, и удельная кинетическая энергия.

\section{4. Анализ решения для поля скоростей}

Из [50] было следует, что решение (7) описывает однонаправленное диффузионное течение (если $\psi$ или $\gamma$ равно нулю) или в противном случае сводится к однонаправленному течению путем поворота горизонтальных осей. При этом каждая из проекций вектора скорости на горизонтальные оси координат не имеет нулевых точек внутри рассматриваемого слоя вязкой жидкости. За счет того, что обе скорости обращаются в нуль на обеих границах слоя, описываемое решением (7) течение является возвратным (рис. 1).

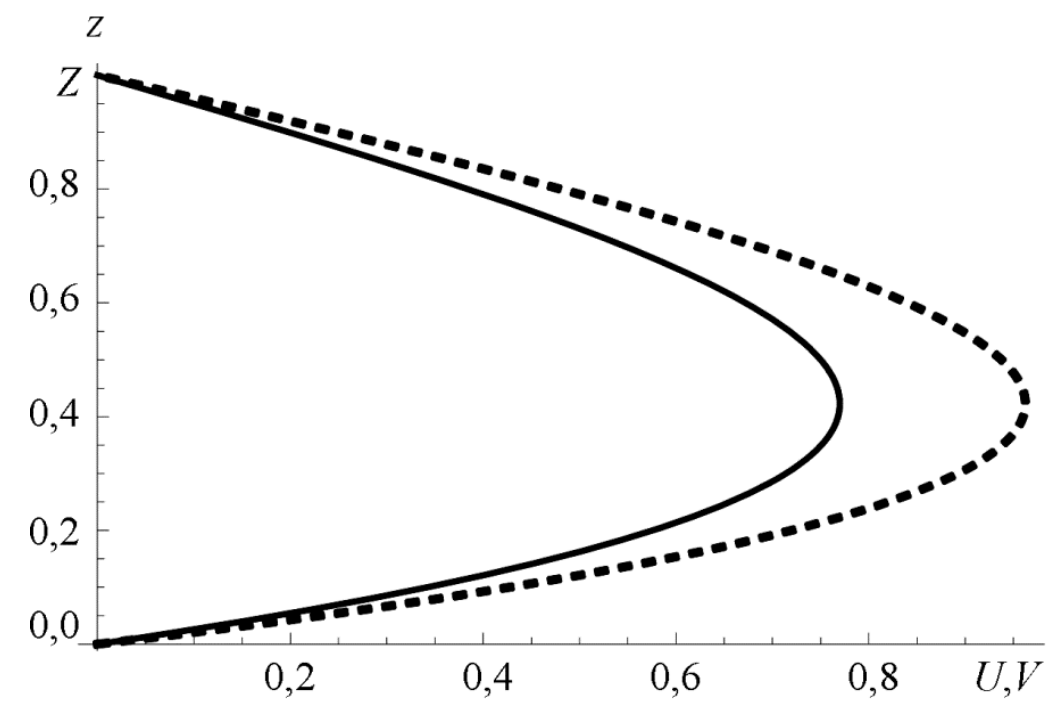

Рис. 1. Профили проекций $U$ (сплошная линия) и $V$ (пунктирная линия) вектора скорости при $\psi=2 ; \gamma=2,5$

В силу указанных обстоятельств получается, что годографом вектора скорости будет отрезок, лежащий на одной из горизонтальных осей (если $\psi$ или $\gamma$ равно нулю) или на прямой общего вида (в противном случае), но в обоих случаях обязательно одним из концов данного отрезка будет начало координат. При этом через любую точку указанного отрезка (кроме второго его конца) годограф проходит дважды (рис. 2). 


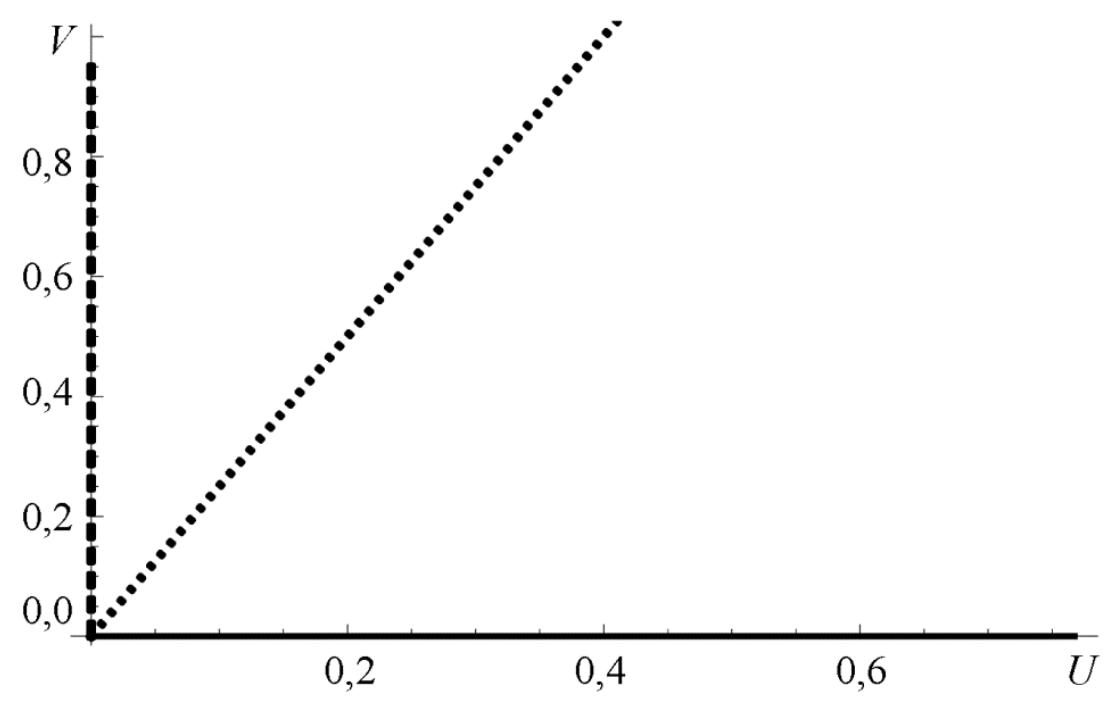

Рис. 2. Годографы вектора скорости при $\psi=2 ; \gamma=0$ (сплошная линия); при $\psi=0 ; \gamma=2,5$ (штриховая линия) и при $\psi=2 ; \gamma=5$ (пунктирная линия)

\section{5. Исследование свойств поля касательных напряжений}

Рассмотрим теперь, какими свойствами обладает поле касательных напряжений, порождаемой скоростями (7). Компоненты тензора касательных напряжений согласно [51-54] определяются по формулам:

$$
\begin{aligned}
\tau_{x y} & =\tau_{y x}=\eta\left(\frac{\partial V_{x}}{\partial y}+\frac{\partial V_{y}}{\partial x}\right) ; \\
\tau_{x z}=\tau_{z x} & =\eta\left(\frac{\partial V_{x}}{\partial z}+\frac{\partial V_{z}}{\partial x}\right) ; \\
\tau_{y z}=\tau_{z y} & =\eta\left(\frac{\partial V_{y}}{\partial z}+\frac{\partial V_{z}}{\partial y}\right),
\end{aligned}
$$

где $\eta$ - коэффициент динамической вязкости. С учетом структуры поля скорости (2) ненулевыми будут только два касательных напряжения:

$$
\begin{aligned}
\tau_{x z} & =\eta \frac{\partial U}{\partial z}=\frac{\eta}{h} \frac{\partial U}{\partial Z}=\frac{\psi \eta}{h}\left(3 Z^{2}-6 Z+2\right) ; \\
\tau_{y z} & =\eta \frac{\partial V}{\partial z}=\frac{\eta}{h} \frac{\partial V}{\partial Z}=\frac{\gamma \eta}{h}\left(3 Z^{2}-6 Z+2\right) .
\end{aligned}
$$

Очевидно, что касательные напряжения (8) обращаются в нуль одновременно, т. е. одновременно меняют тип с растягивающего на сжимающее (или наоборот). Кроме того, это происходит не более двух раз, так как обе функции (8) являются квадратичными параболами. И только одна из этих точек - точка

$$
Z_{*}=\frac{3-\sqrt{3}}{3}=1-\frac{\sqrt{3}}{3} \approx 0,42265-
$$


будет принадлежать рассматриваемому слою жидкости (рис. 3). Эта точка соответствует локальному, экстремуму, который является глобальным на области течения. В точке экстремума, расположенной ближе к нижней границе слоя, скорости жидкости принимают значения:

$$
\begin{gathered}
U\left(Z_{*}\right)=\psi\left(\frac{3-\sqrt{3}}{3}\right)\left(-2+1-\frac{\sqrt{3}}{3}\right)\left(-1+1-\frac{\sqrt{3}}{3}\right)=\psi\left(\frac{3-\sqrt{3}}{3}\right)\left(1+\frac{\sqrt{3}}{3}\right) \frac{\sqrt{3}}{3}= \\
=\psi\left(\frac{3-\sqrt{3}}{3}\right)\left(\frac{3+\sqrt{3}}{3}\right) \frac{\sqrt{3}}{3}=\psi\left(\frac{9-3}{9}\right) \frac{\sqrt{3}}{3}=\frac{2 \sqrt{3}}{9} \psi
\end{gathered}
$$

и соответственно

$$
V\left(Z_{*}\right)=\frac{2 \sqrt{3}}{9} \gamma
$$

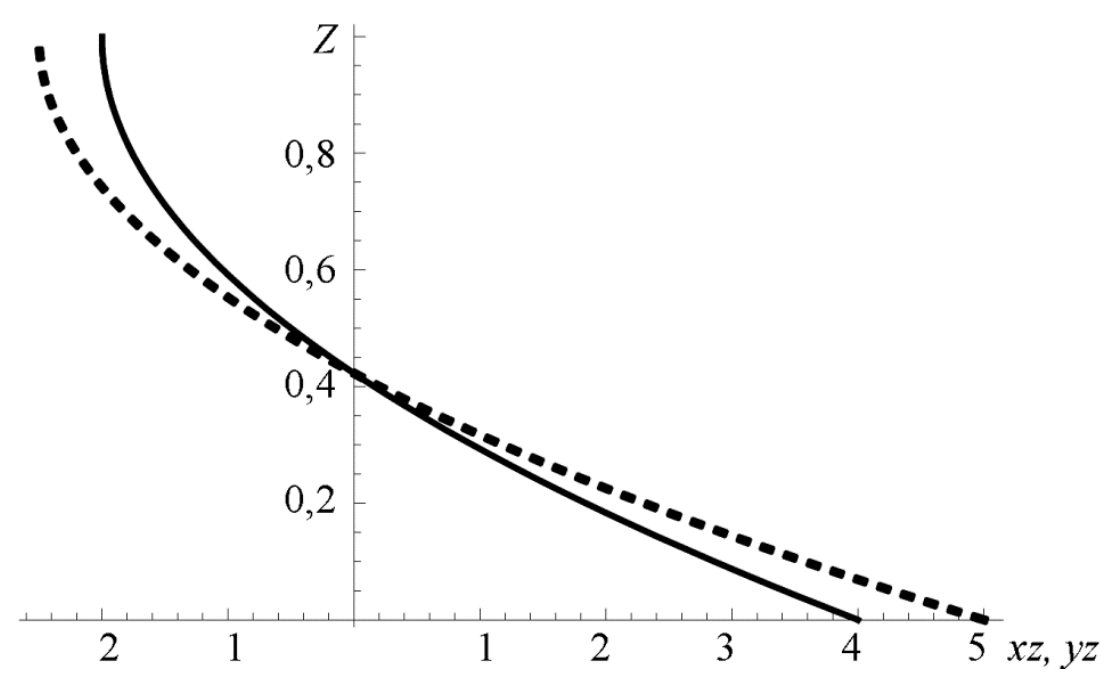

Рис. 3. Профили касательных напряжений $\tau_{x z}$ (сплошная линия) и $\tau_{y z}$ (пунктирная линия)

$$
\text { при } \psi=2 ; \gamma=2,5
$$

Обратим внимание на следующее обстоятельство:

$$
\operatorname{rot} \boldsymbol{V}=\left|\begin{array}{ccc}
\boldsymbol{i} & \boldsymbol{j} & \boldsymbol{k} \\
\frac{\partial}{\partial x} & \frac{\partial}{\partial y} & \frac{\partial}{\partial z} \\
V_{x} & V_{y} & V_{z}
\end{array}\right|=\left|\begin{array}{ccc}
\boldsymbol{i} & \boldsymbol{j} & \boldsymbol{k} \\
\frac{\partial}{\partial x} & \frac{\partial}{\partial y} & \frac{\partial}{\partial z} \\
U & V & 0
\end{array}\right|=\frac{\partial V}{\partial z} \boldsymbol{i}-\frac{\partial U}{\partial z} \boldsymbol{j}=\frac{\tau_{y z}}{\eta} \boldsymbol{i}-\frac{\tau_{x z}}{\eta} \boldsymbol{j} .
$$

Другими словами, ненулевые компоненты вектора завихренности $\boldsymbol{\Omega}=\operatorname{rot} \boldsymbol{V}$ с точностью до постоянного множителя совпадают с касательными напряжениями $\tau_{x z}$ и $\tau_{y z}$. Как было показано выше, в точке $Z_{*}$ оба напряжения одновременно принимают нулевое значение. Это означает, что в подслое $Z_{*}$ течение становится безвихревым (вырождение в потенциальное). 


\section{6. Анализ свойств удельной кинетической энергии}

Удельная кинетическая энергия

$$
E_{k}=\frac{\rho}{2}\left(V_{x}^{2}+V_{y}^{2}\right)=\frac{\rho}{2}\left(U^{2}+V^{2}\right)
$$

очевидно, обращается в нуль только тогда, когда становятся равными нулю обе скорости $U, V$ одновременно. Это происходит только обеих границах рассматриваемого слоя и нигде более. При этом она не равна тождественно нулю. Это означает, что у кинетической энергии имеются точки экстремума. Найдем их:

$$
\frac{d E_{k}}{d Z}=\frac{\rho}{2}\left(2 U \frac{d U}{d Z}+2 V \frac{d V}{d Z}\right)=\rho\left(U \frac{\tau_{x z} h}{\eta}+V \frac{\tau_{y z} h}{\eta}\right)=\frac{\rho h}{\eta}\left(U \tau_{x z}+V \tau_{y z}\right) .
$$

Подставим в правую часть последнего равенства решение (7) для компонент поля скорости и выражения (8) для отвечающих им напряжений:

$$
\frac{d E_{k}}{d Z}=\frac{\rho h}{\eta}\left(\psi^{2}+\gamma^{2}\right) Z^{2}(-2+Z)(-1+Z)\left(3 Z^{2}-6 Z+2\right) .
$$

Таким образом, получаем, что точками экстремума кинетической энергии $E_{k}$ являются либо границы слоя, либо точка

$$
Z_{*}=\frac{3-\sqrt{3}}{3},
$$

где обращаются в нуль оба касательных напряжения (рис. 4).

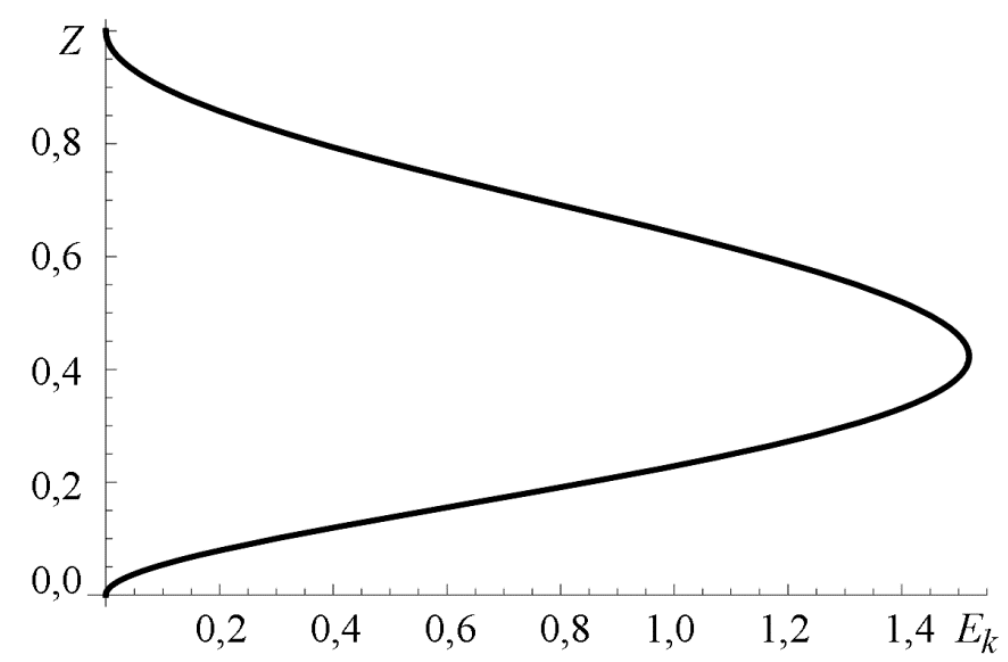

Рис. 4. Профиль кинетической энергии $E_{k}$ при $\psi=2 ; \gamma=2,5$

\section{7. Анализ средней скорости течения}

Одной из характеристик течения помимо проанализированных выше, является его средняя скорость. С точностью до константы, отвечающей площади сечения, через которое проходит жидкость, средняя скорость совпадает с расходом жидкости. 
Расход жидкости $Q_{x}$ в направлении оси $O x$ определяется следующим интегралом:

$$
Q_{x}=\int_{0}^{1} U(Z) d Z=\psi \int_{0}^{1}[Z(-2+Z)(-1+Z)] d Z=\psi \int_{0}^{1}\left[Z^{3}-3 Z^{2}+2 Z\right] d Z=\psi\left[\frac{Z^{4}}{4}-Z^{3}+Z^{2}\right]_{0}^{1}=\frac{\psi}{4}
$$

Аналогичным образом рассчитывается расход жидкости в направлении оси $O y$ :

$$
Q_{y}=\int_{0}^{1} V(Z) d Z=\gamma \int_{0}^{1}[Z(-2+Z)(-1+Z)] d Z=\frac{\gamma}{4}
$$

Таким образом, средняя скорость потока в обоих направлениях оказывается прямо пропорциональна величине горизонтальных градиентов концентрации $a$ и $b$, определяющих значения $\psi$ и $\gamma$.

Заметим, что если в последних двух выражениях положить $\psi=\gamma=0$, расход жидкости в обоих горизонтальных направлениях будет нулевым. Именно ввиду этого обстоятельства построенное решение (7) можно рассматривать как обобщение классического течения Бириха-Остроумова [26, 27]. С другой стороны, в случае $\psi=\gamma=0$ обе проекции вектора скорости, определяемые формулами (7), оказываются тождественно нулевыми и движение жидкости как таковое отсутствует. Таким образом, необходимым условием существования концентрационного течения, отвечающего поставленным краевым условиям (5) и (6), является условие ненулевого расхода жидкости.

\section{8. Заключение}

В статье представлено обобщение течения Бириха-Остроумова на случай неодномерных диффузионных течений при неоднородном распределении поля концентрации на верхней границе рассматриваемого горизонтального слоя. Полученное точное решение описанной краевой задачи неспособно описывать возникновение зон с обратным течением, но отражает наблюдаемый в жидкости эффект попеременного усиления/ослабления скоростей течения в выделенных направлениях. Отличительной особенностью построенного решения является возможность управления числом точек стратификации поля касательных напряжений, определяемых по точному решению для скоростей течения. Максимальное число зон, на которое расслаивается поле напряжений, равно двум. В одной из них напряжение является растягивающим, во второй - сжимающим. Проведенный анализ показал, что если точки стратификации обоих касательных напряжений совпадают, то эта точка будет определять экстремум кинетической энергии.

\section{Литература}

1. Зуев А. Л., Костарев К. Г. Экспериментальное исследование особенностей концентрационно-капиллярной конвекции // Вестник Пермского научного центра УрО РАН. - 2009. № 4. - С. 4-15.

2. Концентрационная конвекция, инициируемая затопленным источником ПАВ / Р. В. Бирих, Е. С. Мазунина, А. И. Мизев, Р. Н. Рудаков // Конвективные течения. - 2009. № 4. - C. 063-084.

3. Евстратова К. И., Купина Н. А., Малахова Е. Е. Физическая и коллоидная химия. М. : Высшая школа, 1990. - 487 с.

4. Петров Н. Х. Исследование процессов выборочной сольватации в бинарных растворителях методами флуоресцентной спектроскопии // Химия высоких энергий. - 2006. Т. 40, № 1. - С. 25-39. 
5. Гуревич А. Е., Капченко Л. Н., Кругликов Н. М. Теоретические основы нефтяной гидрогеологии. - Л. : Недра, 1972. - 272 с.

6. Петров Т. Г., Трейвус Е. Б., Касаткин А. П. Выращивание кристаллов из растворов. Л. : Недра, 1983. - 200 с.

7. Карцев А. А. Гидрогеология нефтяных и газовых месторождений. - 2-е изд., перераб. и доп. - М. : Недра, 1972. - 280 с.

8. Аристов С. Н., Просвиряков Е. Ю., Спевак Л. Ф. Нестационарная слоистая тепловая и концентрационная конвекция Марангони вязкой несжимаемой жидкости // Вычислительная механика сплошных сред. - 2015. - Т. 8, № 4. - C. 445-456. - DOI: 10.7242/19996691/2015.8.4.38.

9. Бесчастнов М. В., Соколов В. М., Кац М. И. Аварии в химических производствах и меры их предупреждения. - М. : Химия, 1976. - 368 с.

10. Пряников В. И. Техника безопасности в химической промышленности. - М. : Химия, 1989. - 288 c.

11. Сургучев М. Л., Желтков Ю. В., Симкин Э. М. Физико-химические микропроцессы в нефтегазоносных пластах. - М. : Недра, 1984. - 215 с.

12. Walmsley H. L. The calculation of the electrostatic potentials that occur when tanks are filled with charged liquids // J. Electostatics. - 1991. - Vol. 26. - P. 201-226. DOI: 10.1016/0304-3886(91)90016-9.

13. Walmsley H. L. Threshold potentials and discharge charge transfers for the evaluation of electrostatic hazards in road-tanker loading // J. Electrostatics. - 1991. - Vol. 26. - P. 157-174. DOI: 10.1016/0304-3886(91)90013-6.

14. Walmsley H. L., Mills J. S. Electrostatic ignition hazards in road tanker loading: Part $1 / /$ J. Electrostatics. - 1992. - Vol. 28. - P. 61-88. - DOI: 10.1016/0304-3886(92)90028-R.

15. Walmsley H. L., Gregory K. E. Electroctatic ignition hazards in road tanker loading: Part 2 // J. Electrostatics. - 1992. - Vol. 28. - P. 99-124. - DOI: 10.1016/0304-3886(92)90065-2.

16. Walmsley H. L., Gregory K. E. Electrostatic ignition hazards in road tanker loading: Part 3 // J. Electrostatics. - 1992. - Vol. 28. - P. 125-48. - DOI: 10.1016/0304-3886(92)90066-3.

17. Fung P., Chen H., Touchard G. G., Radke C. J. A nonlinear corrosion double layer model for laminar flow electrification of hydrocarbon liquids in long metal pipes // J. Electrostatics. 1997. - Vol. 40. - P. 45-54. - DOI: 10.1016/S0304-3886(97)00013-2.

18. Гершуни Г. 3., Жуховицкий Е. М. Конвективная устойчивость несжимаемой жидкости. - М. : Наука, 1972. - 392 с.

19. Рыжков И. И., Степанова И. В. Групповые свойства и точные решения модели вибрационной конвекции бинарной смеси // Прикладная механика и техническая физика. - 2011. Т. 52, № 4. - С. 72-83.

20. Бурмашева Н. В., Просвиряков Е. Ю. Точное решение для установившихся конвективных концентрационных течений типа Куэтта // Вычислительная механика сплошных сред. - 2020. - Т. 13, № 3. - С. 337-349. - DOI: 10.7242/1999-6691/2020.13.3.27.

21. Lin C. C. Note on a class of exact solutions in magneto-hydrodynamics // Arch. Rational Mech. Anal. - 1957. - Vol. 1. - P. 391-395. - DOI: 10.1007/BF00298016.

22. Сидоров А. Ф. О двух классах решений уравнений механики жидкости и газа и их связи с теорией бегущих волн // Прикл. мех. и техн. физ. - 1989. - № 2. - С. 34.

23. Аристов С. Н., Просвиряков Е. Ю., Новый класс точных решений уравнений термодиффузии // Теоретические основы химической технологии. - 2016. - Т. 50, № 3. - С. 294-301. DOI: $10.7868 / \mathrm{S} 0040357116030027$.

24. Burmasheva N. V., Prosviryakov E. Yu. On Marangoni shear convective flows of inhomogeneous viscous incompressible fluids in view of the Soret effect // Journal of King Saud University Science. - 2020. - Vol. 32, iss. 8. - P. 3364-3371. - DOI: 10.1016/j.jksus.2020.09.023. 
25. Князев Д. В. Плоские течения вязкой бинарной жидкости между подвижными твердыми границами // Прикладная механика и техническая физика. - 2011. - Т. 52, № 2 (306). С. $66-72$.

26. Остроумов Г. А. Свободная конвекция в условиях внутренней задачи. - М. : Гос. изд-во технико-теорет. лит-ры, 1952. - 256 с.

27. Бирих Р. В. О термокапиллярной конвекции в горизонтальном слое жидкости // ПМТФ. - 1966. - № 3. - С. 69-72.

28. Su T. C. Obtaining the exact solutions of the Navie - Stokes equations // International journal of non-linear mechanics. - 1985. - Vol. 20, No. 1. - P. 9-19.

29. Петров А. Г. Точное решение уравнений Навье-Стокса в слое жидкости между движущимися параллельно пластинами // Прикладная механика и техническая физика. - 2012. T. 53, № 5. - C. 13-18.

30. Пухначев В. В. Симметрии в уравнениях Навье-Стокса // Успехи механики. - 2006. T. 4, № 1. - C. 6-76.

31. Riabouchinsky D. Quelques considerations sur les mouvements plans rotationnels d' un liquide // C. R. Hebdomadaires Acad. Sci. - 1924. - Vol. 179. - P. 1133-1136.

32. Пухначев В. В. Групповые свойства уравнений Навье-Стокса в плоском случае // ПМТФ. - 1960. - № 1. - С. 83-90.

33. Аристов С. Н., Князев Д. В., Полянин А. Д. Точные решения уравнений Навье-Стокса с линейной зависимостью компонент скорости от двух пространственных переменных // Теорет. основы хим. технологии. - 2009. - Т. 43, № 5. - С. 547-566.

34. Просвиряков Е. Ю. Точные решения трехмерных потенциальных и завихренных течений Куэтта вязкой несжимаемой жидкости // Вестник НИЯУ МИФИ. - 2015. - Т. 4, № 6. C. 501-506. - DOI: 10.1134/S2304487X15060127.

35. Polyanin A. D., Zhurov A. I. Integration of linear and some model non-linear equations of motion of incompressible fluids // International Journal of Non-Linear Mechanics. - 2013. Vol. 49. - P. 77-83. - DOI: 10.1016/j.ijnonlinmec.2012.08.004.

36. Ludlow D. K., Clarkson P. A., Bassom A. Nonclassical symmetry reductions of the threedimensional incompressible Navier-Stokes equations // Journal of Physics A General Physics. 1999. - Vol. 31, No. 39. - 7965. - DOI: 10.1088/0305-4470/31/39/012.

37. Аристов С. Н., Полянин А. Д. Точные решения трехмерных нестационарных уравнений Навье-Стокса // Доклады АН. - 2009. - Т. 427, № 1. - С. 35-40.

38. Пухначев В. В. Симметрии в уравнениях Навье-Стокса // Успехи механики. - 2006. № 6. - С. 3-76.

39. Кузнецова Ю. Л., Скульский О. И., Пышнограй Г. В. Течение нелинейной упруговязкой жидкости в плоском канале под действием заданного градиента давления // Вычислительная механика сплошных сред. - 2010. - Т. 3, № 2. - С. 55-69.

40. Аристов С. Н., Скульский О. И. Точное решение задачи течения шестиконстантной модели жидкости Джеффриса в плоском канале // Прикл. мех. и технич. физика. - 2002. Т. 43, № 6. - С. 39-45.

41. Князев Д. В., Колпаков И. Ю. Точные решения задачи о течении вязкой жидкости в цилиндрической области с меняющимся радиусом // Нелинейная динамика. - 2015. - Т. 11, № 1. - С. 89-97.

42. Гончарова О. Н., Резанова Е. В., Люлин Ю. В., Кабов О. А. Изучение конвективных течений жидкости и спутного потока газа с учетом испарения // Теплофизика высоких темпеpaтур. - 2017. - T.55, № 6. - C. 720-732. - DOI: 10.7868/S0040364417060072.

43. Бурмашева Н. В., Просвиряков Е. Ю. Крупномасштабная слоистая стационарная конвекция вязкой несжимаемой жидкости под действием касательных напряжений на верхней 
границе. Исследование поля скоростей // Вестн. Сам. гос. техн. ун-та. Сер. Физ.-мат. науки. 2017. - T. 21, № 1. - C. 180-196. - DOI: 10.14498/vsgtu1527.

44. Burmasheva N. V., Prosviryakov E. Yu. Temperature field investigation in layered flows of a vertically swirling viscous incompressible fluid under two thermocapillar forces at a free boundary // Diagnostics, Resource and Mechanics of materials and structures. - 2019. - Iss. 1. - P. 6-42. DOI: 10.17804/2410-9908.2019.1.006-042.

45. Burmasheva N. V., Larina E. A., Prosviryakov E. Yu. Unidirectional convective flows of a viscous incompressible fluid with slippage in a closed layer // AIP Conference Proceedings. 2019. - Vol. 2176. - 030023. - DOI: 10.1063/1.5135147.

46. Бурмашева Н. В., Просвиряков Е. Ю. Термокапиллярная конвекция вертикально завихренной жидкости // Теоретические основы химической технологии. $-2020 .-$ Т. 54, № 1. C. 114-124. - DOI: 10.31857/S0040357119060034.

47. Бурмашева Н. В., Просвиряков Е. Ю. Точное решение уравнений Навье-Стокса, описывающее пространственно неоднородные течения вращающейся жидкости // Труды института математики и механики УрО РАН. - 2020. - Т. 26, № 2. - С. 79-87. DOI: 10.21538/0134-4889-2020-26-2-79-87.

48. Burmasheva N. V., Larina E. A., Prosviryakov E. Yu. Unidirectional convective flow of viscous incompressible fluid in a closed horizontal layer with the perfect slip condition // AIP Conference Proceedings. - 2020. - Vol. 2315. - 020010. - DOI: 10.1063/5.0036714.

49. Burmasheva N. V., Privalova V. V., Prosviryakov E. Y. Layered Marangoni convection with the Navier slip condition // Sādhanā. - 2021. - Vol. 46 (1). - 55. - DOI: 10.1007/s12046-02101585-5.

50. Burmasheva N. V., Prosviryakov E. Yu. Analysis of non-one-dimensional shear concentration convective flows of a viscous incompressible fluid in a plane horizontal layer with motionless boundaries // AIP Conference Proceedings. - 2020. - Vol. 2315. - 020007. - DOI: 10.1063/5.0036710.

51. Седов Л. И. Механика сплошной среды. Том 1. - М. : Наука, 1970. - 492 с.

52. Трусделл К. Первоначальный курс рациональной механики сплошных сред. - М. : Наука, 1975. - 592 с.

53. Валландер С. В. Лекции по гидроаэромеханике. Учеб. пособие. - Л. : Изд-во Ленингр. ун-та, 1978. - 296 с.

54. Покровский В. Н. Статистическая механика разбавленных суспензий. - М. : Наука, 1978. - $136 \mathrm{c}$. 